\title{
Resiliency in Medical Students and Residents Participating in the Global Health AMPATH Kenya Elective
}

\author{
Courtney Leigh Keilman and Jenny Baenziger, MD \\ Indiana University School of Medicine and Indiana University Center for Global \\ Health
}

Burnout has become a prominent issue among healthcare providers. The demands of working long shifts, prolonged periods of stress and increased time spent charting all contribute to this phenomenon. Current research suggests that an intrinsic sense of resilience may be key in combating this epidemic. Just as our sense of empathy and compassion can be cultivated through experience, so too can our level of resilience. We propose that engaging in an international health project during medical professional education may promote a greater sense of resiliency. To analyze this, we will use the Connor-Davidson Resilience Scale $\odot$ to survey $4^{\text {th }}$ year medical students and residents at the Indiana University School of Medicine (IUSM) that have participated in the AMPATH rotation at Moi University in Kenya between June 2018 and May 2019. To determine the potential change in resilience we will ask our subjects to complete the survey prior to their trip, as well as 1-months and 1-year after their trip. This study may lead to the advocacy for such projects to be integrated into medical education curricula to combat the growing problem that is physician burnout. 